\title{
Factors That Affect the Rehabilitation Duration in Patients With Congenital Muscular Torticollis
}

\author{
Ah Young Jung, MD, Eun Young Kang, MD, Sung Hoon Lee, MD, \\ Doo Hyeon Nam, MD, Ji Hwan Cheon, MD, Hyo Jung Kim, MD
}

Department of Rehabilitation Medicine, Kwangju Christian Hospital, Gwangju, Korea

\begin{abstract}
Objective To determine which factors affect the rehabilitation duration in patients with congenital muscular torticollis (CMT) and to predict the duration of rehabilitation and prognosis.

Methods One hundred and eighteen patients (79 males and 39 females) who were diagnosed with CMT and received physical therapy were enrolled in this study. We retrospectively reviewed the information in terms of sex, gestational age, birth weight, methods of delivery, fetal presentation, age at diagnosis, the affected sternocleidomastoid (SCM) muscle site, SCM muscle thickness, ratio of muscle thickness on the affected side to that on the unaffected side (called the 'abnormal/normal $[\mathrm{A} / \mathrm{N}]$ ratio'), and range of motion for cervical rotation and side bending.

Results The SCM muscle thickness and A/N ratio had a positive linear relationship with the rehabilitation duration. Patients who were in the breech position needed longer rehabilitation. The birth weight and age at diagnosis were negatively correlated with the rehabilitation duration. However, the cervical range of motion, mass site, sex, gestational age, and methods of delivery were not correlated with the rehabilitation duration.

Conclusion Patients with a thicker SCM, lower birth weight, and history of breech delivery had a longer rehabilitation duration.
\end{abstract}

Keywords Congenital torticollis, Prognosis, Factors

\section{INTRODUCTION}

Congenital muscular torticollis (CMT) is caused by shortening or contracture of the sternocleidomastoid

Received May 19, 2014; Accepted September 3, 2014

Corresponding author: Eun Young Kang

Department of Rehabilitation Medicine, Kwangju Christian Hospital, 37 Yangnim-ro, Nam-gu, Gwangju 503-715, Korea

Tel: +82-62-650-5165, Fax: +82-62-671-5775, E-mail: eykang74@hanmail.net

(c) This is an open-access article distributed under the terms of the Creative Commons Attribution Non-Commercial License (http://creativecommons. org/licenses/by-nc/3.0) which permits unrestricted noncommercial use, distribution, and reproduction in any medium, provided the original work is properly cited.

Copyright $\odot 2015$ by Korean Academy of Rehabilitation Medicine
(SCM) muscle. The head of the patient tilts toward the contractured muscle, and the chin rotates to the opposite direction. CMT is the third most common musculoskeletal disorder, followed by congenital hip dislocation and congenital talipes equinovarus [1]. Its incidence varies from $0.017 \%$ to $1.9 \%$ [2-4]. Because CMT is associated with cranial and facial asymmetry, plagiocephaly, or scoliosis due to the asymmetric cervical position, its early detection and treatment are considered to be crucial [5].

CMT is classified as SCM tumor, muscular torticollis, and positional torticollis: SCM tumor demonstrates a palpable mass within the SCM, muscular torticollis does not demonstrate any palpable mass within the stiff SCM, 
and positional torticollis demonstrates an abnormal posture without a palpable mass or stiff SCM muscle. SCM tumor of infancy appears within 2-4 weeks after birth and gradually disappears by 4 to 8 months. It is located in the middle of the SCM or the lower third of the sternalis and has a fusiform shape. A relatively well-demarcated, non-cystic intramuscular mass with homogeneous echogenicity is noted on ultrasonography [6].

Conservative therapy is considered as the first line treatment and it includes stretching the shortened SCM, rotating the head toward the shortened SCM, and tilting it in the opposite direction while the shoulders are fixed. It is important to start the treatment as early as possible. Non-surgical treatments provide good outcomes in most cases; however, surgical intervention can be performed in patients with continuous loss of range of motion, those who show failure of conservative treatment, or those with progressive maxillofacial asymmetry. The severity of torticollis and delay in starting the treatment can negatively affect the surgical outcome $[7,8]$.

It is not surprising that many studies show that early treatment provides a positive outcome during the course of CMT; however, the exact prognostic factors remain unclear. Therefore, this study investigated the factors that affect the prognosis of CMT patients.

\section{MATERIALS AND METHODS}

This study was approved by the Institutional Review Board of the Kwangju Christian Hospital and did not require consent of the patients or their parents because it was a retrospective study that used data from the medical records. Out of the 149 infants who visited the hospital for abnormal neck posture from December 2008 to December 2012, 31 infants who had other neurodevelopmental disorders that could have caused the abnormal neck posture, who had ocular torticollis, who did not undergo an ultrasound examination, and who were unable to receive a follow-up after the diagnosis at the hospital were excluded, and finally, 118 infants with CMT (79 males and 39 females) were enrolled in this study.

Detailed medical history taking, physical examinations, and radiography were performed in the subjects. Information on the risk factors associated with the medical history was also collected, and other accompanying symptoms, such as plagiocephaly and facial asymmetry, and developmental hip dysplasia were observed. Plagiocephaly means an asymmetrical head shape with more than $5 \mathrm{~mm}$ diagonal difference, the difference between two diagonal cranial diameters, $30^{\circ}$ from the midsagittal plane of the skull [9]. Facial asymmetry means that the patients have a smaller chin, flatter zygomatic arch, and/ or flatter forehead on the affected side compared to the unaffected side on inspection. Developmental hip dysplasia was diagnosed by hip ultrasound, as the patient was placed in the lateral decubitus position, the $\alpha$ angle corresponding to the inclination of the bony acetabulum and the $\beta$ angle corresponding to the inclination of the cartilaginous acetabulum on a coronal view of the hip joint were measured, and then less than $60^{\circ}$ of the $\alpha$ angle and more than $45^{\circ}$ of the $\beta$ angle [10].

The location of the lesion and mass in the SCM was confirmed via physical examination, and an experienced physiatrist examined the range of motion of the neck for determining any limitation in movement. The ultrasound machine used in this study was the $9 \mathrm{MHz}$ linear trans-
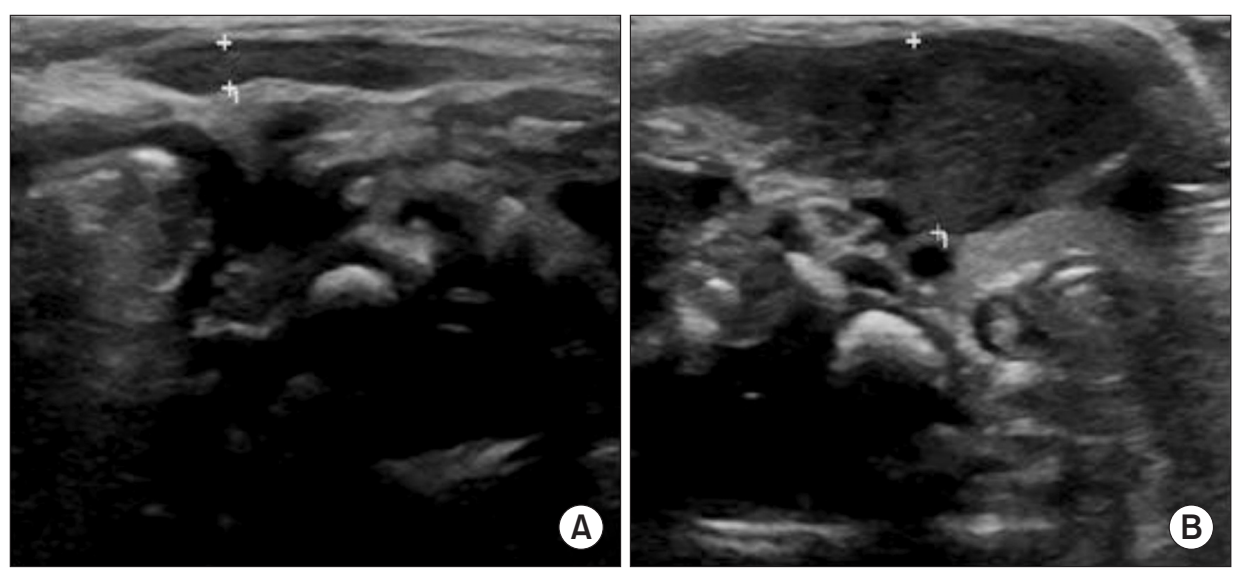

Fig. 1. Ultrasonographic findings of the sternocleidomastoid muscle in patients with congenital muscular torticollis. (A) Unaffected side and (B) affected side measurements at the thickest point in the longitudinal view. 
ducer (LOGIQ 9, Milwaukee, WI, USA) designed by GE Healthcare, and an experienced radiologist confirmed the difference between the thickness and shape of bilateral SCM muscles. The subjects were asked to lie on their back (supine position) for the test. The neck was extended slightly, and they were asked to look in the opposite direction. The thickness of the thickest SCM muscle fiber was measured. The thickness of SCM on the affected side was compared with that on the unaffected side (Fig. 1).

For physiotherapy, the neck was bent in the direction opposite to that of the affected side and rotation exercise was performed on the affected side, twice a week for 30 minutes each. The parents of the subjects were educated to help their children perform continuous passive joint exercise and active exercise. When the difference in the ranges of motion on both sides was less than $5^{\circ}$, the treatment was considered to be successful and physiotherapy was discontinued.

Statistical analysis was performed using the SPSS ver. 18.0 for Windows (SPSS Inc., Chicago, IL, USA) software program. The Student t-test was used to analyze the following factors: sex, location of lesion, methods of delivery, and accompanying defects; and a Pearson correlation test was used to analyze the following factors: age, birth weight, difference in the thickness of SCM on the affected side and that on the unaffected side, ratio of the muscle thickness on the affected side to that on the unaffected side, and range of motion of passive cervical movement. The results were considered statistically significant when the $\mathrm{p}$-value was less than 0.05 .

\section{RESULTS}

There were more number of males than females among the 118 subjects who were diagnosed with CMT and the sex ratio was found to be $79: 39$ ( $66.9 \%$ vs. $33.1 \%)$. The torticollis affected side was the right side in 57 subjects (48.3\%) and the left side in 61 subjects $(51.7 \%)$, which was not significantly different. Also, 88 subjects (74.6\%) were delivered vaginally, and 30 subjects $(25.4 \%)$ were delivered via cesarean section. In terms of the fetal presentation, 98 subjects (83.0\%) were in a cephalic position, and 20 subjects (17.0\%) were in a breech position.

The mean age at diagnosis was $68.80 \pm 61.37$ days, the mean gestational age was $39.19 \pm 1.40$ weeks, and the mean birth weight was $3.22 \pm 0.39 \mathrm{~kg}$. The mean cervi- cal rotation at diagnosis was $73.46^{\circ} \pm 14.19^{\circ}$, and the difference in cervical rotation between both sides was $24.72^{\circ} \pm 13.79^{\circ}$. The mean cervical side-bending at diagnosis was $34.15^{\circ} \pm 9.25^{\circ}$, and the difference in cervical sidebending between both sides was $13.61^{\circ} \pm 9.20^{\circ}$. The mean thickness of the SCM, as confirmed by an ultrasound examination, was $1.00 \pm 0.30 \mathrm{~cm}$ on the affected side, and the ratio of muscle thickness on the affected side to that on the unaffected side (i.e., the $\mathrm{A} / \mathrm{N}$ ratio) was $2.02 \pm 0.84$ (Table 1).

There was no statistically significant difference in the rehabilitation duration based on sex or the torticollis affected side. The mean rehabilitation duration of $138.15 \pm 98.04$ days in patients who were born via normal spontaneous vaginal delivery (NSVD) was shorter than that of $162.00 \pm 123.01$ days in patients who were born via

Table 1. Demographic factors of the CMT patients in this study (n=118)

\begin{tabular}{lc}
\hline & Value \\
\hline Sex & $79(66.9)$ \\
\hline Male & $39(33.1)$ \\
\hline Female & \\
\hline Torticollis affected side & $57(48.3)$ \\
\hline Right & $61(51.7)$ \\
\hline Left & \\
\hline Methods of delivery & $88(74.6)$ \\
\hline NSVD & $30(25.4)$ \\
\hline Cesarean section & \\
\hline Fetal presentation & $98(83.0)$ \\
\hline Cephalic & $20(17.0)$ \\
\hline Breech & $68.80 \pm 61.37$ \\
\hline Age at diagnosis (day) & $3.22 \pm 0.39$ \\
\hline Birth weight $(\mathrm{kg})$ & $39.19 \pm 1.40$ \\
\hline Gestational age $($ wk) & $73.46 \pm 14.19$ \\
\hline Cervical rotation $\left({ }^{\circ}\right)$ & $24.72 \pm 13.79$ \\
\hline Difference in cervical rotation $\left({ }^{\circ}\right)$ & $34.15 \pm 9.25$ \\
\hline Cervical side-bending $\left({ }^{\circ}\right)$ & $13.61 \pm 9.20$ \\
\hline Difference in cervical side-bending $\left({ }^{\circ}\right)$ & $1.00 \pm 0.30$ \\
\hline SCM thickness $(\mathrm{cm})$ & $2.02 \pm 0.84$ \\
\hline Abnormal/normal ratio & \\
\hline Values are presented as number $(\%)$ & \\
\hline
\end{tabular}

Values are presented as number (\%) or mean \pm standard deviation.

CMT, congenital muscular torticollis; NSVD, normal spontaneous vaginal delivery; SCM, sternocleidomastoid. 
Table 2. Comparison of the rehabilitation duration according to the demographic factors

\begin{tabular}{|lcc}
\hline & $\begin{array}{c}\text { Rehabilitation } \\
\text { duration (day) }\end{array}$ & p-value \\
\hline Sex & & 0.678 \\
\hline Male (n=79) & $147.05 \pm 104.68$ & \\
\hline Female (n=39) & $138.48 \pm 106.51$ & \\
\hline Torticollis affected side & & 0.577 \\
\hline Right (n=57) & $138.61 \pm 89.17$ & \\
\hline Left (n=61) & $149.45 \pm 118.25$ & \\
\hline Methods of delivery & & 0.284 \\
\hline NSVD & $138.15 \pm 98.04$ & \\
\hline Cesarean section & $162.00 \pm 123.01$ & \\
\hline Fetal presentation & & $0.039^{*}$ \\
\hline Cephalic & $136.13 \pm 101.49$ & \\
\hline Breech & $190.47 \pm 114.05$ & \\
\hline DDH & & 0.373 \\
\hline Yes (n=10) & $218.00 \pm 142.81$ & \\
\hline No (n=108) & $167.96 \pm 104.51$ & \\
\hline Facial asymmetry & & 0.330 \\
\hline Yes (n=48) & $151.35 \pm 117.28$ & \\
\hline No (n=70) & $131.62 \pm 90.17$ & \\
\hline Plagiocephaly & & 0.287 \\
\hline Yes (n=49) & $138.15 \pm 98.04$ & \\
\hline No (n=69) & $162.00 \pm 123.01$ & \\
\hline
\end{tabular}

Values are presented as mean \pm standard deviation.

NSVD, normal spontaneous vaginal delivery; DDH, developmental dysplasia of the hip.

${ }^{*} \mathrm{p}<0.05$, Student $\mathrm{t}$-test.

cesarean section, but the difference was not significant. The mean rehabilitation duration in patients with a cephalic fetal presentation was $136.13 \pm 101.49$ days, which was shorter than that of $190.47 \pm 114.05$ days in patients with a breech fetal presentation $(\mathrm{p}<0.05)$ (Table 2$)$.

Fourteen subjects experienced a difficult birth or early membrane rupture; 5 subjects, oligohydramnios; 1 subject, clavicle fracture; and 10 subjects (8.5\%), developmental dysplasia of the hip, 49 subjects $(41.5 \%)$ had plagiocephaly, and 48 (40.6\%) had facial asymmetry, but these factors did not show a significant correlation with the rehabilitation duration (Table 2).

The subjects whose condition was detected early and who had a lower birth weight had a longer rehabilitation duration $(\mathrm{p}<0.05)$ (Table 3$)$. There was no statistically significant correlation between the gestational age and the
Table 3. Relationship between the general characteristics and the rehabilitation duration

\begin{tabular}{lcl}
\hline & $\begin{array}{c}\text { Correlation } \\
\text { coefficient }\end{array}$ & p-value \\
\hline Age at diagnosis & -0.388 & $0.000^{* *}$ \\
Gestational age & -0.108 & 0.247 \\
Birth weight & -0.191 & $0.040^{*}$ \\
Cervical rotation & -0.110 & 0.238 \\
Cervical side-bending & 0.037 & 0.691 \\
\hline SCM thickness & 0.237 & $0.010^{*}$ \\
\hline Abnormal/normal ratio & 0.293 & $0.000^{* *}$ \\
\hline
\end{tabular}

SCM, sternocleidomastoid.

${ }^{*} \mathrm{p}<0.05,{ }^{* *} \mathrm{p}<0.01$, Pearson correlation.

rehabilitation duration.

Cervical rotation or side-bending was also not significantly correlated with the rehabilitation duration. But, the subjects with a thicker SCM and a higher $\mathrm{A} / \mathrm{N}$ ratio had a longer rehabilitation duration $(\mathrm{p}<0.05)$ (Table 3$)$.

\section{DISCUSSION}

The mass in the SCM appears at 1-2 weeks after birth and grows in the first 2-4 weeks, but it shrinks and finally disappears in the next 3-6 months. Surgical treatment is required for a mass non-responsive to 4-6 months of conservative treatment or is diagnosed after 1 year of birth [11]. Binder et al. [1] reported incidences of $45.9 \%$ for continuous maxillofacial asymmetry, $24.7 \%$ for intermittent head torticollis, $5.8 \%$ for scoliosis, and $11.6 \%$ for functional asymmetry in patients after the treatment. Canale et al. [12] reported that there was no functional abnormality based on the cervical range of motion, and that the aesthetic problem such as facial asymmetry or head torticollis persisted in $31 \%$ of the patients. Hence, it was considered important to investigate the factors that affect the prognosis of CMT in order to minimize complications and prevent the need for invasive treatment.

The direct cause of CMT is shortening of the muscle due to fibrosis of the SCM, but the cause of muscle fibrosis is unknown [11]. The most commonly suggested mechanisms of such muscle fibrosis are fetal presentation in the uterus and trauma during delivery. Sanerkin and Edwards [13] supported the trauma theory by explaining in their article that bleeding and laceration in the SCM due to trauma during delivery leads to fibrosis. 
However, torticollis also develops in infants who are delivered without a history of traumatic contraction or via cesarean section, which contradicts the birth trauma theory. Moreover, there are no evidences of hematoma or products of bleeding in histological findings of fibrous tissue [14]. The tilted cervical position of the fetus in the uterus may cause shortening of the SCM. The incidence of torticollis in healthy newborns is only $1.5 \%-7 \%$, whereas the incidence of torticollis following a difficult delivery or a breech delivery is $17 \%-40 \%$ [15], and these findings are partly consistent with the present study result which confirmed a longer rehabilitation duration in subjects who were delivered in a breech position. This result was attributed to the severity of the symptoms based on the thickness of the SCM and the A/N ratio, which were $0.98 \pm 0.31 \mathrm{~cm}$ and $1.97 \pm 0.86$, respectively, in the healthy subjects compared to $1.10 \pm 0.20 \mathrm{~cm}$ and $2.25 \pm 0.74$, respectively, in the subjects delivered in a breech position. Other congenital musculoskeletal abnormalities, such as congenital talipes equinovarus and hip dysplasia, support the aforementioned theory of fetal presentation in the uterus. In this study, $25.4 \%$ of the subjects had a gestational history of oligohydramnios, a difficult delivery, gestational diabetes, or clavicle fracture. This study confirmed that the subjects with a lower birth weight had a significantly longer rehabilitation duration. The theoretical evidence or the previous study result that supports this finding was not confirmed, but underweight subjects seemed to have a longer rehabilitation duration because of their underdeveloped muscles as a result of an immature musculoskeletal system.

Kim and Kim [11] suggested that CMT occurs more often on the right side because the right venous pressure is higher than the left venous pressure. Coventry and Harris [3] reported that the posture of the fetus in the uterus may cause torticollis and the incidence of torticollis is two-fold higher on the right side when the baby is in a breech position. Chang et al. [16] also reported that the incidence of torticollis is higher on the right side. In the present study, however, the torticollis affected side did not show a significant difference among the subjects (right $48.3 \%$ and left 51.7\%). Kim et al. [17] reported that the incidence of torticollis was significantly higher in boys than in girls, which was consistent with the result of this study which indicated an almost two times higher incidence in boys ( 79 boys and 39 girls). However, sex and torticollis affected side did not show a significant correlation with the rehabilitation duration.

Many previous studies reported a significant correlation between the age at diagnosis and the rehabilitation duration. Kim et al. [17] reported that the starting point for the treatment did not have a significant correlation with the treatment outcome, and Lee et al. [18] reported that older age at the start of the treatment was associated with a poorer treatment outcome, which was based on their study results which showed successful treatment outcomes in $90.9 \%$ of 88 cases treated within six months, $78.6 \%$ of cases treated within six months to one year, and $50 \%$ of cases treated after one year. Binder et al. [1] reported that $69.3 \%$ of the subjects treated up to 12 months recovered completely, whereas 12 out of the 20 subjects whose treatment started after they turned one year old showed response to conservative treatment but eight subjects underwent surgical treatment. However, Emery [15] reported that the starting point of the treatment in subjects younger than two years of age did not affect the rehabilitation duration, and the results of their study were interpreted to indicate that the subjects who had severe clinical symptoms such as a severely limited cervical range of motion or a mass in the SCM visited the hospital earlier than those who did not have severe clinical symptoms. In this study, the rehabilitation duration was longer when the diagnosis was made earlier. The severity of the disease seemingly affected the age upon hospital admission. The age upon hospital admission was classified into 'within 30 days after birth,' 'between 31 and 60 days after birth,' and ' 61 or more days after birth,' and the thicknesses of the SCM and the A/N ratios in the groups were compared. The mean thickness of the SCM and the $\mathrm{A} / \mathrm{N}$ ratio in the subjects who were admitted to the hospital within 30 days after their birth were $1.14 \pm 0.19 \mathrm{~cm}$ and $2.47 \pm 0.71$, respectively; the mean thickness of the SCM and the $\mathrm{A} / \mathrm{N}$ ratio in the subjects admitted to the hospital between 31 and 60 days after their birth were $1.17 \pm 0.23$ $\mathrm{cm}$ and $2.34 \pm 0.79$, respectively; and the mean thickness of the SCM and the A/N ratio in the subjects admitted to the hospital at 61 or more days after their birth were $0.75 \pm 0.25 \mathrm{~cm}$ and $0.34 \pm 0.50$, respectively. These results were interpreted to indicate that the subjects with severe clinical symptoms were admitted to the hospital earlier than those without severe clinical symptoms $(\mathrm{p}<0.01)$.

The cervical range of motion in CMT is measured based 
on rotation and side bending for making the clinical diagnosis. Lee et al. [6] and Cheng et al. [7] considered that the measurement of cervical rotation was more reliable than that of side bending in terms of the inter-examiner reliability, and they used the cervical rotation angle to predict the rehabilitation duration. In their studies, subjects with a more limited cervical rotation angle had a longer rehabilitation duration than those who had a normal cervical rotation angle. Emery [15] reported a strong correlation between the cervical rotation angle and the side bending angle, but only the cervical rotation angle showed a significant correlation with the rehabilitation duration. In this study, the subjects were divided into three groups based on their cervical rotation and side bending angles, and the matching rate between the side bending angle and the cervical rotation angle was only $52.73 \%$; hence, it was considered difficult to indicate that the cervical rotation angle represents the range of motion. Therefore, both the cervical rotation and side bending angles were measured to investigate their correlation with the rehabilitation duration. The results showed no significant correlation between both the cervical rotation and side bending angles and the rehabilitation duration.

Lee et al. [6] reported that both the thickness of the SCM and the A/N ratio did not show a significant correlation with the rehabilitation duration, when $54 \mathrm{CMT}$ subjects were classified into three groups based on the thickness of the SCM and the A/N ratio for investigating their correlation with the rehabilitation duration in each group. Han et al. [19] reported a significant difference between the thickness of the SCM on the affected side and that on the unaffected side in 46 CMT patients and 6 patients who required surgery or botulinum toxin A injection. Kim et al. [17] investigated the correlation of SCM ultrasound results with the treatment outcome in $52 \mathrm{CMT}$ patients when they were classified into 5 groups depending on the severity of their disease according to the echogenicity and presence of a mass based on the ultrasound results and their reported significant correlations. Han et al. [20] reported that the thickness of the SCM did not affect the rehabilitation duration because the thickness itself was inconsistent according to the age of the patients or their muscle development, but the ratio of the bilateral SCM muscle thickness affected the rehabilitation duration. In this study, both the thickness of the SCM and the $\mathrm{A} / \mathrm{N}$ ratio showed a significant correlation with the reha- bilitation duration. As such, patients with a thicker SCM and a higher $\mathrm{A} / \mathrm{N}$ ratio are deemed to require intensive treatment and a longer rehabilitation duration than average torticollis patients. Also, the treatment compliance can be increased by providing more accurate information regarding the rehabilitation duration to the parents.

The limitations of this study include treatment at home, because exercise was only performed through parent training, which might have caused a difference in treatment outcomes. In further studies, the treatment environment should be controlled for obtaining accurate study results. Also, the severity of the disease depending on the echogenicity and presence of a mass in the ultrasound findings was not investigated in this study, but it can be assessed in future studies.

CMT requires early detection and treatment to prevent sequelae of the limited cervical range of motion and secondary musculoskeletal deformities, such as craniofacial asymmetry, scoliosis, and malocclusion.

This study confirmed the factors that affect the treatment outcome in patients with CMT and their correlation with the rehabilitation duration. The results showed that patients with a thicker SCM, a lower birth weight, and a history of breech delivery had a longer rehabilitation duration.

On the other hand, sex, torticollis affected side, methods of delivery, developmental dysplasia of the hip, facial asymmetry, plagiocephaly, gestational age, and cervical range of motion did not show a significant correlation with the rehabilitation duration.

In conclusion, the thickness of the SCM, A/N ratio, birth weight, and a history of breech delivery were considered to be the factors that significantly affect the rehabilitation duration for CMT.

\section{CONFLICT OF INTEREST}

No potential conflict of interest relevant to this article was reported.

\section{REFERENCES}

1. Binder H, Eng GD, Gaiser JF, Koch B. Congenital muscular torticollis: results of conservative management with long-term follow-up in 85 cases. Arch Phys Med Rehabil 1987;68:222-5. 
2. Cheng JC, Au AW. Infantile torticollis: a review of 624 cases. J Pediatr Orthop 1994;14:802-8.

3. Coventry MB, Harris LE. Congenital muscular torticollis in infancy; some observations regarding treatment. J Bone Joint Surg Am 1959;41A:815-22.

4. Suzuki S, Yamamuro T, Fujita A. The aetiological relationship between congenital torticollis and obstetrical paralysis. Int Orthop 1984;8:175-81.

5. Yu CC, Wong FH, Lo LJ, Chen YR. Craniofacial deformity in patients with uncorrected congenital muscular torticollis: an assessment from three-dimensional computed tomography imaging. Plast Reconstr Surg 2004;113:24-33.

6. Lee JY, Koh SE, Lee IS, Jung H, Lee J, Kang JI, et al. The cervical range of motion as a factor affecting outcome in patients with congenital muscular torticollis. Ann Rehabil Med 2013;37:183-90.

7. Cheng JC, Tang SP, Chen TM. Sternocleidomastoid pseudotumor and congenital muscular torticollis in infants: a prospective study of 510 cases. J Pediatr 1999;134:712-6.

8. Park JM, Choi JH, Lee YH. Ultrasonographic measurement of the sternocleidomastoid muscle in congenital muscular torticollis. J Korean Acad Rehabil Med 1998;22:955-9.

9. Kim SY, Park MS, Yang JI, Yim SY. Comparison of helmet therapy and counter positioning for deformational plagiocephaly. Ann Rehabil Med 2013;37:785-95.

10. Graf R. Classification of hip joint dysplasia by means of sonography. Arch Orthop Trauma Surg 1984; 102:248-55.

11. Kim MO, Kim SJ. Results of the conservative manage- ment of congenital muscular torticollis. J Korean Acad Rehabil Med 1992;16:42-50.

12. Canale ST, Griffin DW, Hubbard CN. Congenital muscular torticollis: a long-term follow-up. J Bone Joint Surg Am 1982;64:810-6.

13. Sanerkin NG, Edwards P. Birth injury to the sternomastoid muscle. J Bone Joint Surg Br 1966;48:441-7.

14. Lee SJ, Han JD, Lee HB, Hwang JH, Kim SY, Park MC, et al. Comparison of clinical severity of congenital muscular torticollis based on the method of child birth. Ann Rehabil Med 2011;35:641-7.

15. Emery C. The determinants of treatment duration for congenital muscular torticollis. Phys Ther 1994; 74:921-9.

16. Chang PY, Tan CK, Huang YF, Sheu JC, Wang NL, Yeh ML, et al. Torticollis: a long-term follow-up study. Zhonghua Min Guo Xiao Er Ke Yi Xue Hui Za Zhi 1996;37:173-7.

17. Kim SJ, Park EM, Choi WK, Seo KS, Yoon JS, Lee EH. The Correlation between outcome and ultrasonographic findings in congenital muscular torticollis. J Korean Acad Rehabil Med 2001;25:601-8.

18. Lee YT, Jahng JS, Park BM. A clinical study of congenital muscular torticollis. J Korean Orthop Assoc 1986;21:423-32.

19. Han JD, Kim SH, Lee SJ, Park MC, Yim SY. The thickness of the sternocleidomastoid muscle as a prognostic factor for congenital muscular torticollis. Ann Rehabil Med 2011;35:361-8.

20. Han SJ, Shin BM, Lee JM, Yoon TS. Factors affecting rehabilitation outcome of congenital muscular torticollis. J Korean Acad Rehabil Med 2010;34:643-9. 